\title{
Prospettiva e rifugio nella pandemia, ovvero il paesaggio visto come uccelli.
}

Fabio Di Carlo

Sapienza Università di Roma, DiAP, Dipartimento di Architettura e progetto fabio.dicarlo@uniroma1.it

\begin{abstract}
Se ogni attività progettuale presuppone un quadro di riferimento -stabile o di previsione e visione- nel quale inscrivere ogni prefigurazione, lo stato di conoscenze e mutazioni sul futuro post-pandemico non è chiaro, né lo sono le necessità e i processi utili per affrontarle. Anche rispetto al ruolo del progetto di paesaggio è possibile organizzare solo risposte parziali e transitorie, che sono forse più prese di coscienza che figurazioni, o che possono essere parte di un ragionamento che ponga nuovi e diversi elementi di orientamento. Nella convinzione che questa fase non possa essere trattata né con strumenti consolidati, né con un processo unitario, questo scritto è volutamente frammentario. Ė quasi una raccolta non ordinata di riflessioni su alcuni aspetti che pongono in relazione il lavoro sul progetto di paesaggio con l'attuale condizione. Qui paesaggio e giardino sono visti come condizioni di prospettiva e rifugio, secondo la teoria di Jay Appleton sull'esperienza del paesaggio, che ci è utile come riferimento.
\end{abstract}

\section{Keywords}

Spazio pubblico, paesaggio urbano, semantica dei luoghi, prospettiva-rifugio.

\begin{abstract}
If every design activity presupposes a framework of reference -stable or of forecast and vision- in which to inscribe every prefiguration, the state of knowledge and mutations on the post-pandemic future is not clear, nor are the needs and processes useful to face them. Even with respect to the role of the landscape architecture it is possible to organize only partial and transitory responses, which are perhaps more awareness than figurations, or which may be part of a reasoning that poses new and different elements of orientation. Convinced that this phase cannot be dealt with either with consolidated instruments, or with a unitary process, this paper is deliberately fragmentary. It is almost an unordered collection of reflections on some aspects that relate the work on the landscape project to the current condition. Here landscape and garden are seen as conditions of perspective and refuge, according to Jay Appleton's theory on landscape experience, which is useful for us as a reference.
\end{abstract}

\section{Keywords}

public space, urban landscape, semantics of places, prospect-refuge. 


\section{Silenzio operoso}

Al netto dei temi fondamentali di sopravvivenza, l'emergenza sanitaria attuale ha posto tutti i progettisti in una condizione interrogativa sul significato dei propri interessi, sull'impatto che pandemia e post-pandemia avranno su questi e, all'inverso, di come la propria azione possa portare un contributo positivo nel breve, medio e lungo periodo. C'è stato molto 'rumore', ma anche un corpo di riflessioni utili, che mi piace definire 'silenzio operoso', sugli oggetti di studio e soprattutto sull'organizzazione del pensiero che si rende necessario per comporre risposte a domande mai formulate e per inquadrarle secondo prospettive mai esplorate.

È un interrogativo che coinvolge tutte le discipline, progettuali e non, e in particolare quelle del progetto di paesaggio. Nella condizione di confinamento, paesaggio, giardino e spazio pubblico hanno rappresentato qualcosa di duplice, tra desiderio e rischio, tra prospettiva individuale di fuga e di vita all'esterno, ma anche necessità di rifugio e protezione, in maniera simile alla teoria del prospect-refuge formulata da Jay Appleton in The Experience of Landscape (1975) e ripresa nell'ambito degli studi dell'ecologia cognitiva da Almo Farina (2007) o in quelli di teorie dell'architettura, da Annemarie Dosen e Michael Ostwald (2013). Appleton, pur considerando alcune tesi dell'etologia limitati- ve rispetto alla dimensione culturale del paesaggio, muoveva dai risultati degli studi sui comportamenti animali, per introdurre alcune interessanti omologie tra umani e animali, gli uccelli in particolare, che possono risultare utili come similitudine in questo discorso.

La pandemia è per la sua immediatezza e prossimità spazio-temporale ancor più violenta dell'attenzione con cui ci si pongono le emergenze climatiche ed ecologiche. Le ricadute di queste ultime sono inesorabili ma più dilatate nel tempo, e si lavora su queste con metodiche ormai abbastanza chiare. Con il COVID-19 tutte le emergenze sono passate immediatamente in secondo piano, tranne quando vedevamo acque e cieli più azzurri, che ci hanno reso tutti più consapevoli di alcuni problemi che si rendevano ancor più evidenti. Ma queste immagini non ci davano risposte immediate alla contingenza. Come in ogni crisi, da un lato è necessario porre attenzione a possibili derive, ma è altrettanto utile aprire i sensi a ciò che ci offrono in termini di possibilità. Matteo Meschiari, antropologo, in un'intervista rilasciata a Doppiozero, alla domanda se la cultura umanistica possa dare un contributo alla crisi attuale, definisce il virus come un "dinosauro nero non estetizzabile", che ci impone di abbandonare i soggetti usuali della rappresentazione e di conquistare nuovi strumenti cognitivi: 
Non ho alcun dubbio sul fatto che la cultura umanistica potrà e dovrà assumere un ruolo guida nell'attraversamento delle molte crisi che ci attendono. Faccio un esempio: il romanzo. Da circa trent'anni l'editoria e un pubblico abitudinario di lettori hanno premiato il romanzo neoliberista, [...]. Covid-19 è entrato in queste rappresentazioni e le ha svuotate. [...] adesso nel salotto di nonna è arrivato un tremendo dinosauro nero, prelinguistico, prerazionale, sordo alla dialettica dei filosofi, non estetizzabile dai poeti. [...] Forse la gente non vorrà guardare negli occhi il dinosauro nero, ma avrà comunque bisogno di strumenti cognitivi per riflettere sulla nuova realtà, e non più in termini di svago ma di sopravvivenza. (Meschiari, 2020)

Sarà stata di certo la paura di questo dinosauro nero, più profonda di quelle comuni, che rende difficile parlare dei riflessi del COVID-19 rispetto al progetto paesaggio, e che mi ha fatto preferire un silenzio pressoché assoluto. Quando ho cercato di razionalizzare l'origine di questo mio disagio e difficoltà di espressione, ne ho focalizzato almeno due aspetti. Il primo disagio era relativo al contesto linguistico nel quale si potevano esporre delle riflessioni. Come parlare di futuro dei paesaggi umani in un momento in cui ha dominato una dizione come quella di 'distanziamento sociale', che ferisce come un orrendo ossimoro? Tutto ciò che è sociale prende forma all'interno di un concetto di collettività, vicinanza e relazione. Forse già 'distanziamento fisico' sarebbe stato meno fastidioso e al contempo preciso. II secondo disagio derivava dall'osservazione di un rincorrersi di proposte, talvolta frutto di esigenze di mediatizzazione personale e spesso prive di senso critico quanto di ironia. Tutte all'insegna del ridisegno di nuovi modelli di spazio solo apparentemente distanti da quanto si è fatto negli ultimi cento anni, ma in realtà meccanicisticamente determinati dalle stesse forme di pensiero. Tutte disegnate a partire da modi di pensare precedenti, elaborate dagli stessi software e algoritmi costruiti tra modernità e postmodernità, senza considerare che le indicazioni su una contin-

\section{Giardinieri in politica: Oudolf e Clément}

Tra le molte riflessioni emerse c'è ovviamente un ragionamento sul portato politico del progetto di paesaggio, che non è una novità, ma che rilega pensieri diversi, che muovono da Pierre Grimal, attraverso Yves Lacoste, fino ai diversi scritti di Marco Martella, nella sua rivista Jardins o nel suo secondo romanzo Giardini in tempo di guerra, dove il protagonista lascia la devastazione della guerra in Bosnia e percorre una fuga salvifica attraverso alcuni giardini d'Europa. Durante il primo confinamento tutti siamo stati testimoni della forza con la quale la biologia del mondo riemergeva a livello globale, con implicazioni importanti rispetto a paesaggio e ambiente, come sui risvolti etici del rapporto tra politica, neoliberismo e condizione di crisi. Alcuni paesaggisti, in particolare dei giardinieri, hanno espresso posizioni molto particolari.

Piet Oudolf ha trascorso il confinamento inondando le pagine dei social con i suoi giardini. Non soltanto le foto e il bellissimo film Five Seasons, ma moltissimi dei suoi disegni, anche tecnici, scansionati e diffusi quasi come una lezione universale sul suo modo di fare giardini. II 17 agosto il Magazine di Hauser \& Wirth, per i quali Oudolf ha realizzato un field a Somerset, ha pubblicato un'intervista di Francis Till all'autore (Till, 2020). Interrogato esplicitamente su quale sia il ruolo del giardino nella condizione anomala del confinamento, le risposte di Oudolf appaiono semplici, quasi banali, e volutamente circoscritte a questioni molto vicine a quelle dei giardini terapeutici. La sua riflessione sui 'giardini al tempo della pandemia' parte con lo spunto di una domanda sul ruolo del colore nel suo lavoro, che viene da lui ribaltata in una direzione più ampia sul ruolo del giardino:

"Gardens are not only for people. [...] There are so many more creatures that can enjoy what we are doing there, and you can see that. And then when the flowers have gone, then you get plants that have seeds, which is another source for birds to come to the garden. So I think the garden benefits a lot more than just people." 
Quindi Oudolf definisce l'artificio del giardino come azione umana destinata a forme di fruizione e benefici estesi molto oltre la sfera umana. Fare giardini e paesaggi, costruire nature, in questo senso diviene il riconoscimento di una necessità che attraverso l'atto culturale di rappresentazione della natura si estende alla costruzione di un giardino-mondo con finalità che vanno ben oltre le necessità umane. Alla domanda se la pandemia possa al contrario riportare le persone verso una postura più antropocentrica, Oudolf risponde:

\begin{abstract}
"Yes, and I think that's probably why outdoor space is so important for people, because as you can imagine so many people live in cities with no garden, and they need an escape. And not only the beaches or a big walk in the woods are an escape".
\end{abstract}

Analogamente indiretta è la risposta alla domanda sul ruolo del tempo nei suoi giardini:

\begin{abstract}
"I'd say plants are just what they are, and they show what they do through the seasons. What is important is that we learn to look at and understand our environment and do better for what we see around us. We can become so focused on what we have to do that we forget what we can do and what we probably really want to do. I think that gardens are a good metaphor for change..."
\end{abstract}

Gilles Clément è stato invece più esplicitamente polemico rispetto all'esercizio del potere in questa fase. In una lettera aperta del mese di aprile inserita nella pagina web dell'editore Quodlibet - ma anche tradotta e pubblicata da Enrico Falqui sul sito Landscape First e diversamente articolata per un'intervista su Domusweb - Clément esprime chiaramente alcuni concetti. II primo è l'insostenibilità dell'azione politica diffusa, che si appoggia a una terminologia di guerra come forma di controllo esteso della popolazione, nella difesa prevalente di interessi economici.

Il pericolo imprevedibile - qualunque sia la sua natura - pone il governo in un dovere di controllo assoluto e legittimo con il pretesto di combattere il perico-
Io in questione. Quindi il vocabolario guerriero utilizzato serve per sviluppare una strategia la cui utilità politica è sottomissione. Ė facile guidare un popolo sottomesso, impossibile fare lo stesso con un popolo libero (Clément, 2020).

II secondo è la necessità di un ripensamento generale dei modelli di sviluppo, che coincidono solo parzialmente con i modelli della 'decrescita felice', per appoggiarsi invece a una diversa declinazione delle relazioni cultura-natura in termini di necessità

\begin{abstract}
... se volessimo imparare una lezione dalla tragedia che ci ha colpito attraverso il covid19, essa suggerisce agli uomini e le donne che abitano il Pianeta che un modo 'diverso' di consumare, produrre e vivere in comunità coese, è assolutamente possibile. I potenti di ogni parte del mondo, si opporranno violentemente a questa tendenza [...]. Lo hanno già dimostrato su una scala molto piccola [...]: in Francia, un esercito di poliziotti è stato inviato contro una comunità di agricoltori nel Nord-Est della Francia, denominati gli "zadisti di Notre Dame des Landes" la cui unica colpa non era quella di aver occupato delle terre abbandonate, quanto piuttosto di aver inventato e sviluppato "un'arte di vivere" che utilizza la bio-diversità senza distruggerla attraverso un'economia autosostenibile al difuori dei circuiti di spesa tradizionale. Questa esperienza era divenuta un modello e, per questo motivo, questo incendio ha dovuto essere spento a tutti i costi. Ma il fuoco non è spento. Sta covando sotto la cenere. E per quanto piccolo, può infiammare i continenti nel prossimo futuro. Per salvarli dalla distruzione del mercato e immergerli nella dinamica di una 'Ri-Creazione': quella di imparare nuovamente a vivere. Dovremo mai ringraziare i microrganismi per averci fatto aprire gli occhi? (Clément, 2020).
\end{abstract}

\section{Risemantizzare lo spazio collettivo}

Clément ha ragione nel dire che la politica è ricorsa alle metafore della guerra per sopperire alla difficoltà di gestione del problema della diffusione del COVID-19. Durante il confinamento molti hanno paragonato quella condizione a uno stato di guerra. Molti altri invece, alla ricerca di una normalizzazione almeno apparente, esplicitamente si opponevano a questa terminologia. 


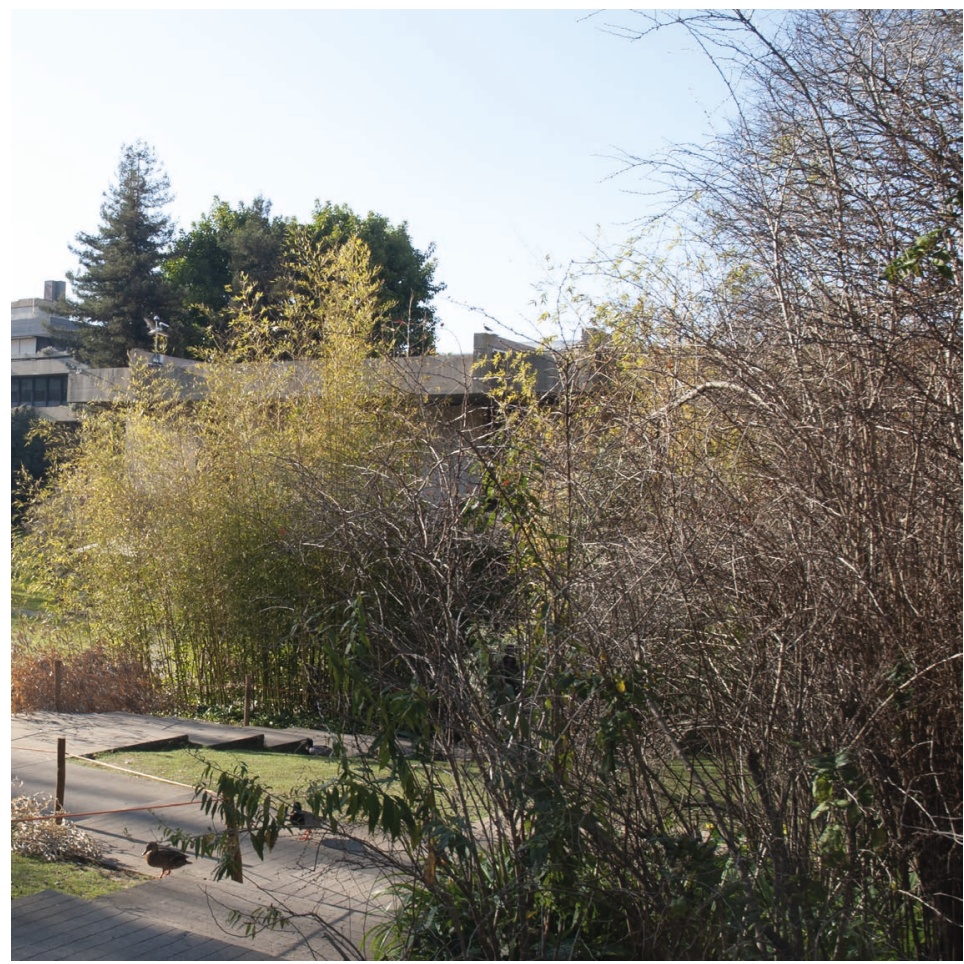

Che molti o pochi abbiamo effettivamente provato questa sensazione non può essere oggetto di questa riflessione, ma di fatto si è creata una dicotomia forte, tra paura e desiderio rispetto ai luoghi che erano parte della nostra quotidianità condivisa.

Ė appropriato dire che oggettivamente i nostri spazi hanno subito una ri-semantizzazione a causa della pandemia. Da un lato il riconoscere allo spazio pubblico, in tutte le sue forme, un nuovo status legato alla condizione di rischio potenziale che deriva dalla sua frequentazione, in particolare quella collettiva. Ma con una pulsione di eguale portata, anche se con postura di segno opposto, tutti hanno sentito una grande necessità di vita in esterni, attribuendo nuovi significati a luoghi secondari o poco vissuti, di prossimità o interni alle abitazioni. Credo quindi che si possa affermare che c'è stato un processo collettivo di riconoscimento di significati nuovi o diversi per molti resse e dalle infrastrutture a questi connesse, verso quelli di prossimità e locali, riproponendo in altre forme e dimensioni scalari il discorso sul policentrismo, più volte discusso nel corso del Novecento e inserito tra le politiche di sviluppo della Commissione europea nell'ambito dello Schema di Sviluppo dello Spazio Europeo (Musterd, Sako \& I, Zelm, 2001).

Ė qui utile riprendere brevemente il discorso del prospect and refuge, prospettiva e rifugio, estendendo il suo significato al riconoscimento di una condizione di rischio, che è soggettiva e temporalmente definita, per l'animale come per l'umano. La posizione privilegiata di controllo del territorio, il "seeing without being seen" di Appleton, coincide con la ricerca o la costruzione del rifugio. II rifugio è contemporaneamente luogo di sicurezza per sfuggire alla predazione, ma anche punto privilegiato di osservazione per definire la propria uscita e proiezione verso l'esterno, per soddisfare le proprie necessità. Quindi il cambiamento di una con- 


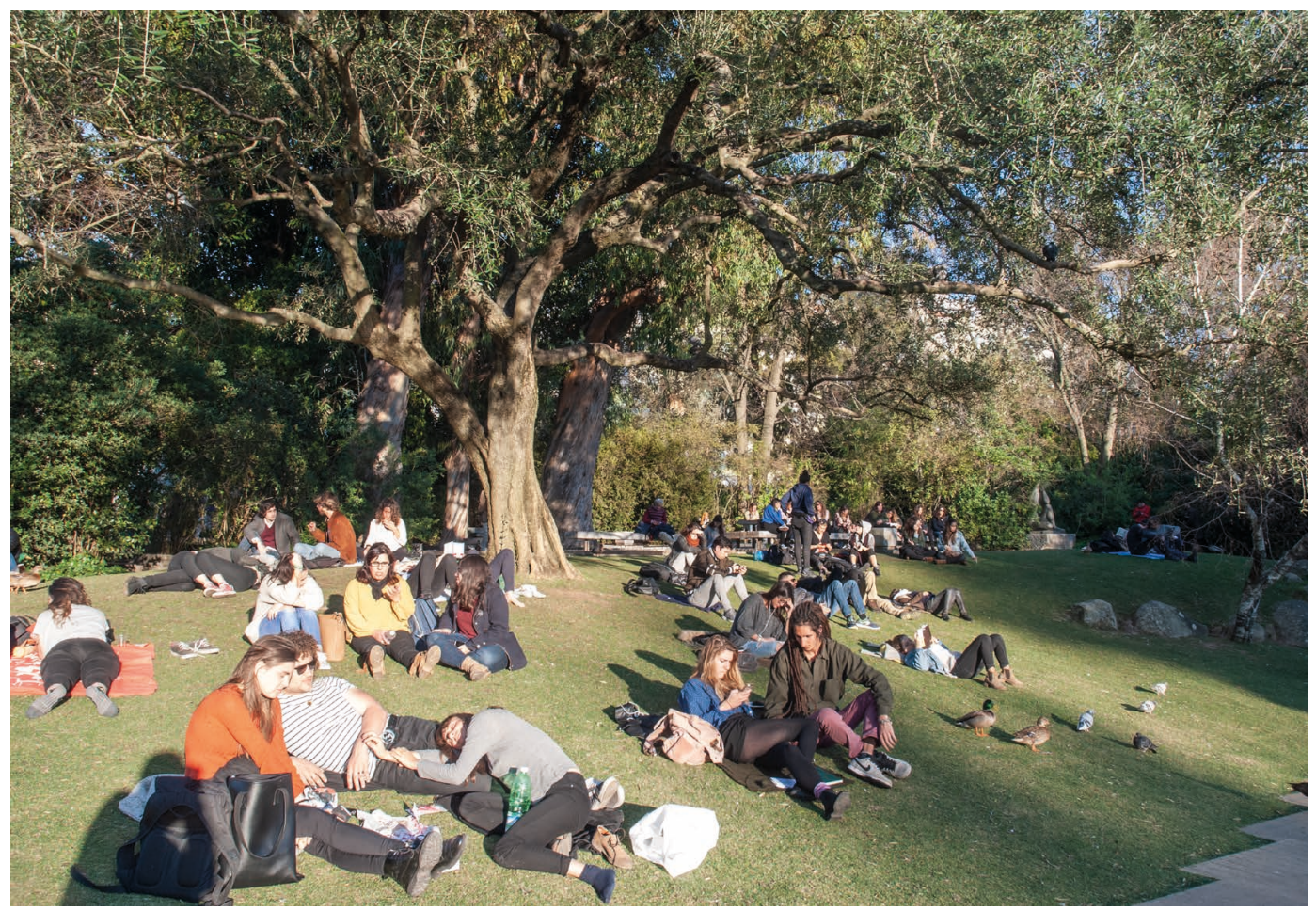

dizione può ribaltare la percezione del proprio habitat e delle sue parti. II rifugio come risposta al rischio e come prospettiva di espansione e di caccia opposta alla posizione di difesa, sono mutevoli, temporanee e scambiabili. In questo senso possono essere interpretate molte delle opzioni sulla progettazione dello spazio di prossimità: "La teoria del prospect and refuge trova una sua spendibilità nell'interpretazione dell'arte, della simbologia ambientale, ma anche nella progettazione del verde pubblico e privato e nelle modalità del suo utilizzo (Farina, 2007).

Questa inversione avviene spesso proprio nei contesti di pericolo temporaneo. Negli scenari di guerre, reali o rappresentate, dove innocenti stupende praterie divengono campi di sangue delle battaglie in campo aperto, o sono cosparse di mine anti-uomo. Nell'immaginario di molti film gli spazi pubblici di città islamiche sono dilaniati da auto-bomba, o come nei film western dove c'è sempre un momento in cui la strada si spopola per lasciar spazio ai pistoleri di fazioni opposte.

Anche in letteratura ci sono diversi passaggi ambientati in luoghi di guerra che propongono una inversione di significati degli spazi di paesaggio. Ne / sentieri dei nidi di ragno di Italo Calvino, ciò si rende evidente. Laddove usualmente la foresta è il luogo insicuro per eccellenza contrapposto invece alla sicurezza dello spazio aperto e soleggiato, nel racconto delle battaglie di Resistenza c'è un'inversione tra 'l'opaco', cioè lo spazio coperto e quasi buio del bosco e del sottobosco che diviene riparo sicuro, contrapposto al rischioso 'aprico', dove si è esposti al nemico, ai cecchini e alle bombe. La luminosità dei campi perde la sua bellezza e genera rischio. Anche briganti e rifugiati nella migliore tradizione letteraria, preferiscono l'opaco come rifugio e ne sopportano volentieri le difficoltà. Analogamente rispetto a epidemie e pandemie, c'è un ampio repertorio di romanzi classici che descrivono città deformate e trasformate da tali eventi. 
Sostanzialmente questa condizione comporta una diversa semantizzazione dei luoghi quotidiani e conosciuti che, istantaneamente, forse temporaneamente o no, acquistano significati di segno opposto sulla base delle condizioni oggettive di prospect and refuge.

\section{Città compatta Vs. sprawl}

Uno dei temi più affrontati in questi mesi ha riguardato la possibilità di aderire a un diverso modello urbano, di fatto proponendo una contrapposizione tra sviluppo per tessuti concentrati e diffusione sul territorio. $\grave{E}$ questo di certo un dibattito lunghissimo, che ha le sue origini in molti testi di Lewis Mumford, che con la sua critica volgeva verso l'utopia di un habitat umano che oggi definiremo tutti come più sostenibile. Un dibattito che periodicamente torna alla ribalta, tra spinte alla densificazione e spraw/ sul territorio, tra limitazione del consumo di suolo e necessità di espansione, tra verticalità e orizzontalità, tra concentrazione urbana e recupero dei centri secondari.

Molti si sono lanciati su idee di ridisegno di nuove forme dell'abitare molto distanti da quanto si è fatto negli ultimi secoli anni in architettura, in urbanistica, nel paesaggio. Anche molti i ballon d'essai di archistar, che memori di alcuni loro miti giovanili lungamente dimenticati e oggi riesumati, vorrebbero spostarci nelle aree interne, tutte da ricostruire nel corpo e nel senso delle riflessioni aperte che pongono.

Tutto il movimento moderno ha ruotato attorno a diversi aspetti di un paradigma di economicità che diventava quasi un'etica. La compattezza esasperata di Peter Oud e del Neufert fortunatamente da noi non ha mai attecchito troppo, ma tutta la cultura razionalista mostrava sempre sé stessa come un virtuosismo nell'ottimizzazione di spazi, tempi e risorse. Pur se ampiamente criticato nelle basi teoriche e come nelle ricadute delle espressioni più radicali, è un paradigma che resta di fatto un dato di confron- zazione delle infrastrutture e del movimento, e molti altri parametri continuano a essere prioritari per ogni progetto.

Del resto, pur se possiamo concordare nel definire la città del Novecento come espressione dello sviluppo capitalistico (Kroll, 1999), di certo non potremmo dirlo di quella medioevale, fatta di reciprocità e contatto molto stretti, o di quella rinascimentale, già più distanziata forse proprio anche come risposta alle pandemie medioevali, secondo le osservazioni di Giancarlo De Carlo (già appropriatamente richiamate in un altro saggio di $A$. Metta in questo volume), o delle trasformazioni e sanificazioni delle città borghesi dell'Ottocento, che introducono diversi elementi volti esplicitamente al miglioramento delle condizioni sanitarie. Di certo le declinazioni smart e sustainable non contribuiranno da sole a ridurre la distanza di identità tra evoluzione urbana e abitanti (La Cecla, 2015).

Probabilmente è prematuro affermare che dovremo ripensare in tutto o in parte, il sistema di funzionamento delle società umane, e degli spazi esterni o interni che viviamo. Analogamente rispetto ai mezzi di movimento e della quantità e qualità degli spostamenti. Vicinanza e assembramento sono concetti di base delle comunità. In quasi tutte le culture la condizione di densità e di concentrazione è da sempre stata garanzia di sopravvivenza e di protezione del gruppo. E anche se i fenomeni dell'urbanesimo dell'Antropocene ci hanno mostrato di recente tutte le deformazioni del sistema, il loro ribaltamento totale ci sembra improbabile e, aggiungo, non necessariamente auspicabile, se non con termini e margini che sono in corso di definizione. Michel Grove, sulle pagine di The Dirt di ASLA, espone un'idea esattamente opposta alla dispersione. Motiva l'affermazione che non siano le forme delle città, compatte o disperse, a determinare variabili dei livelli di rischio di contagi, presenti e futuri, quanto piuttosto altri fattori che rimandano anche qui alla dimensione politica del fare città. 


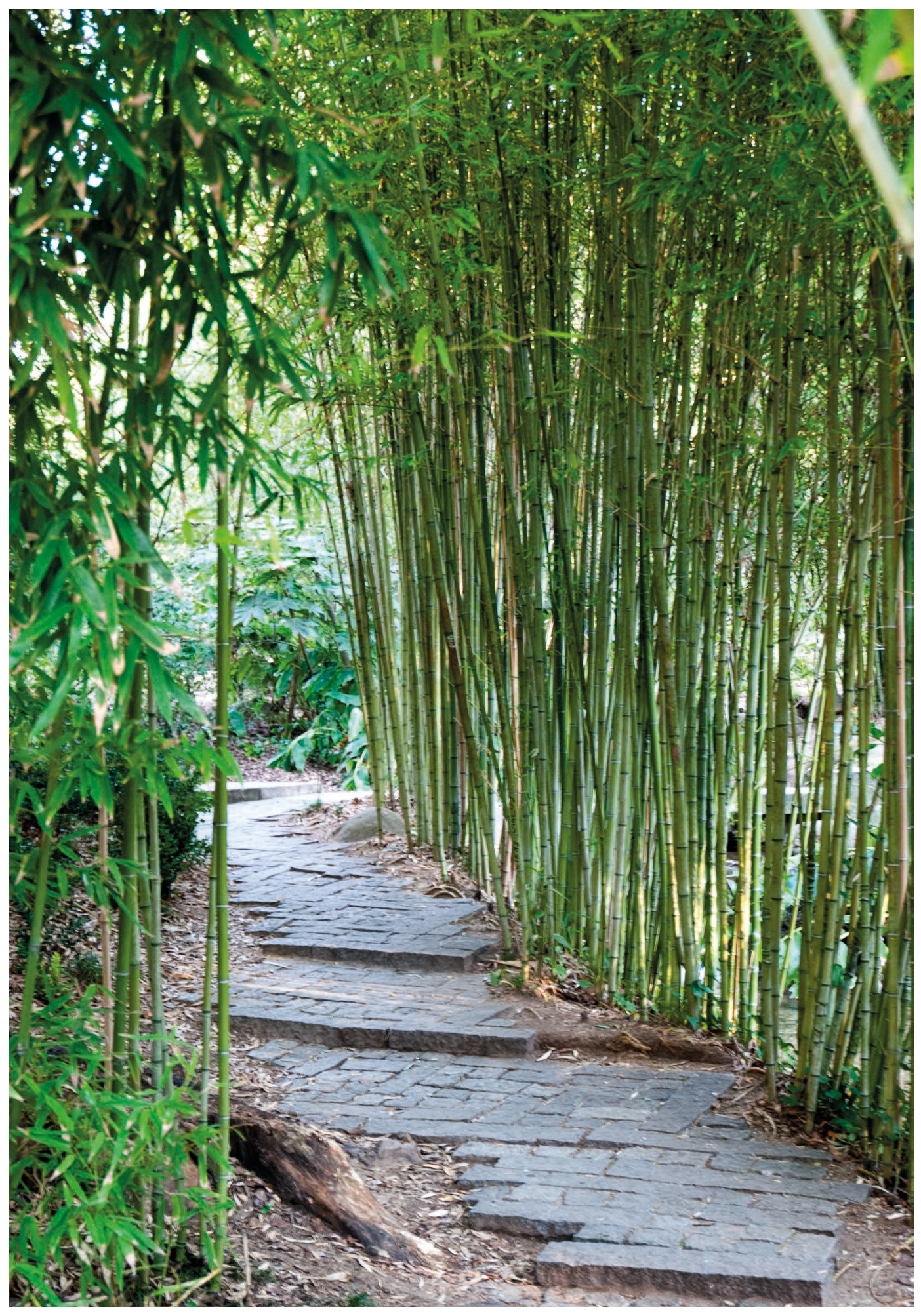




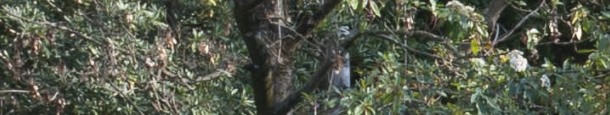

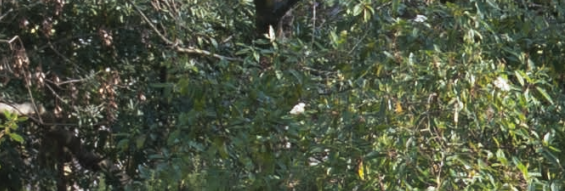

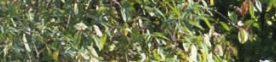

2.

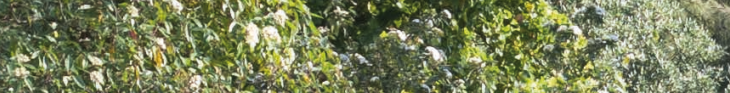

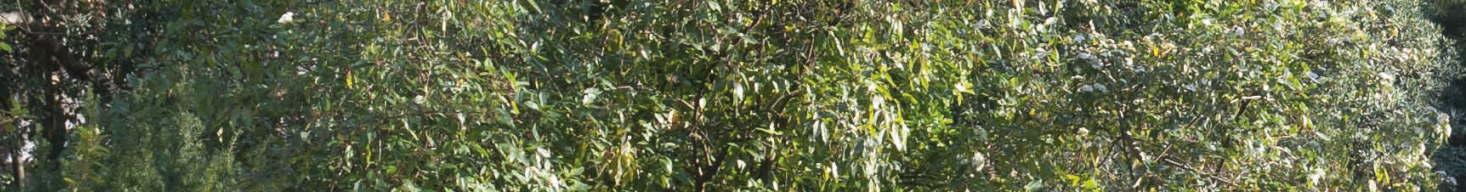

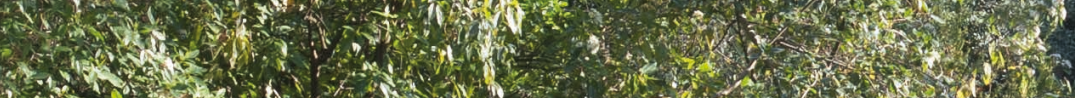

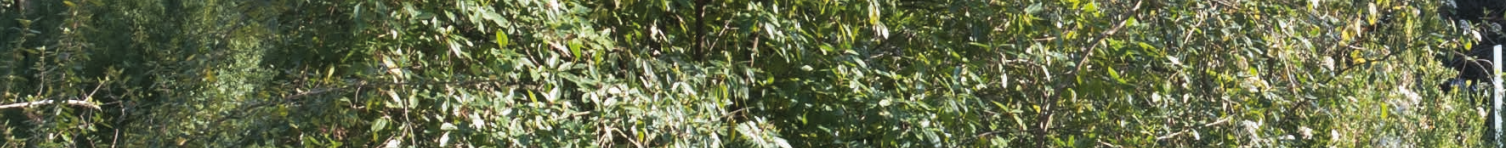
a - at

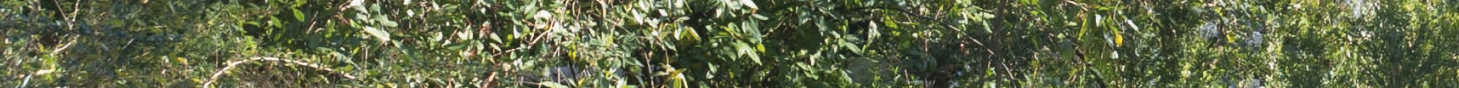

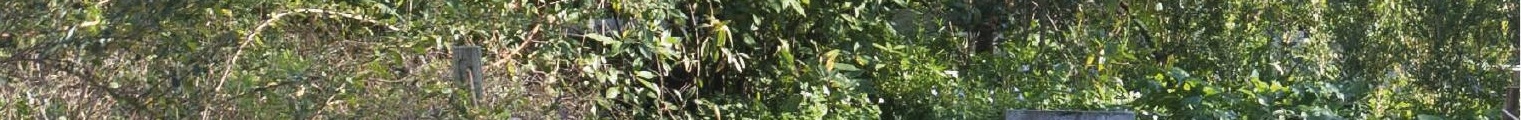
1.

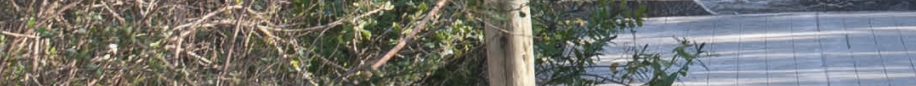

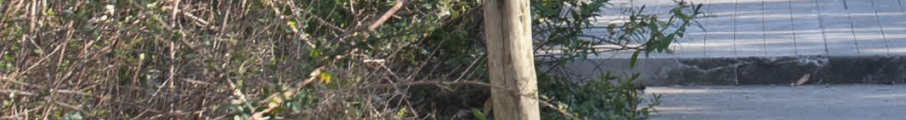

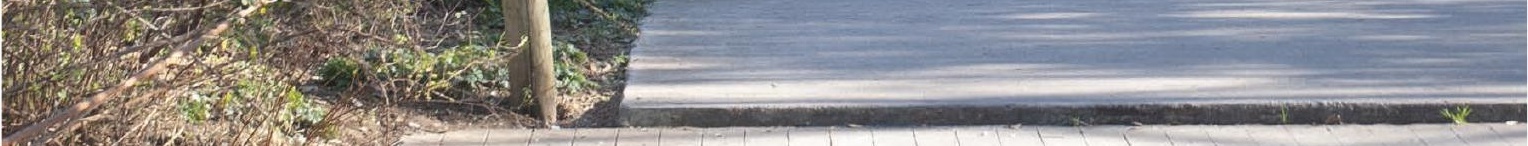

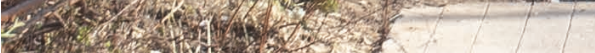

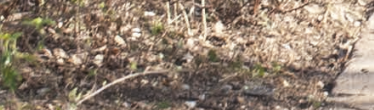

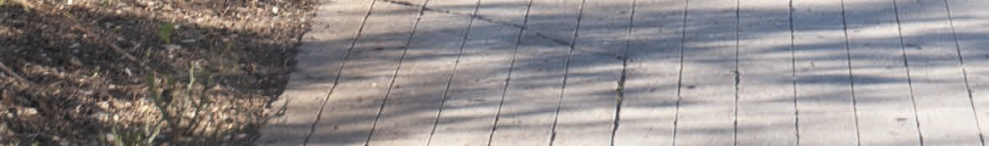

as 


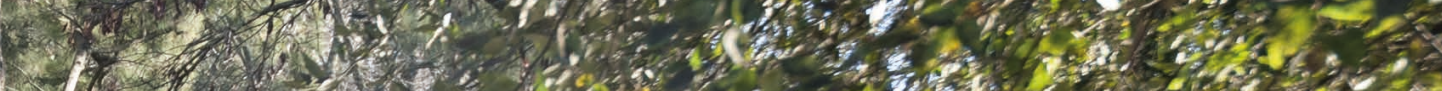

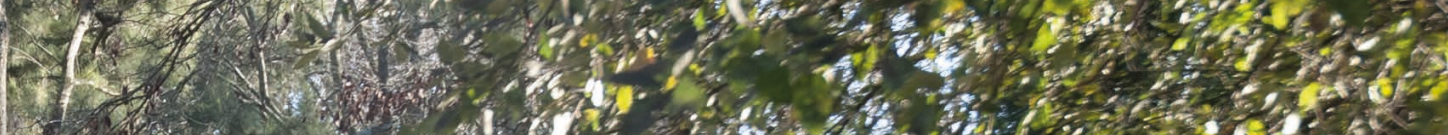
17 H.

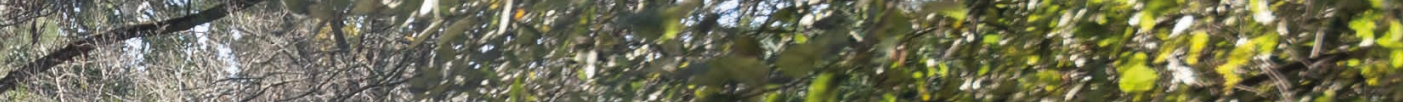

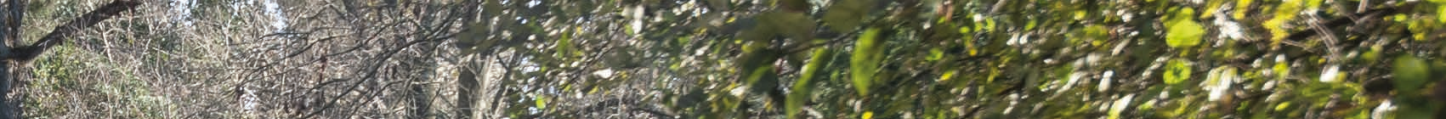

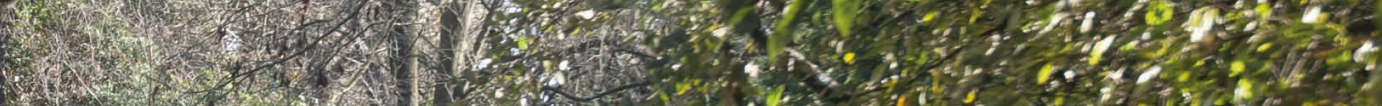

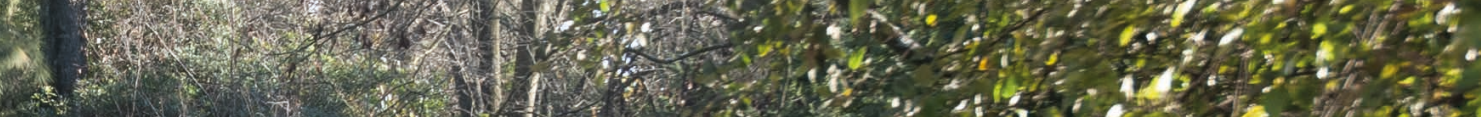

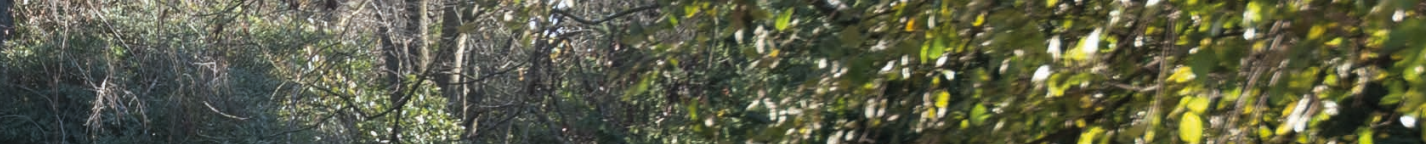

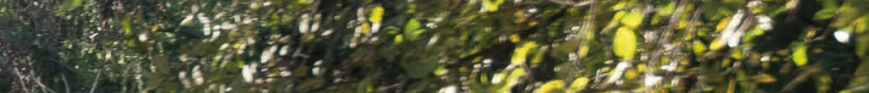

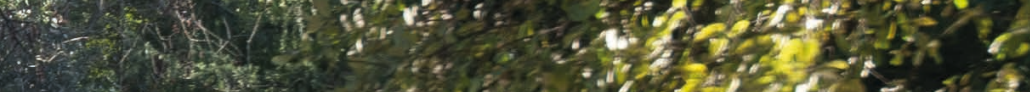

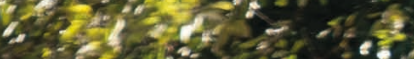

(3)

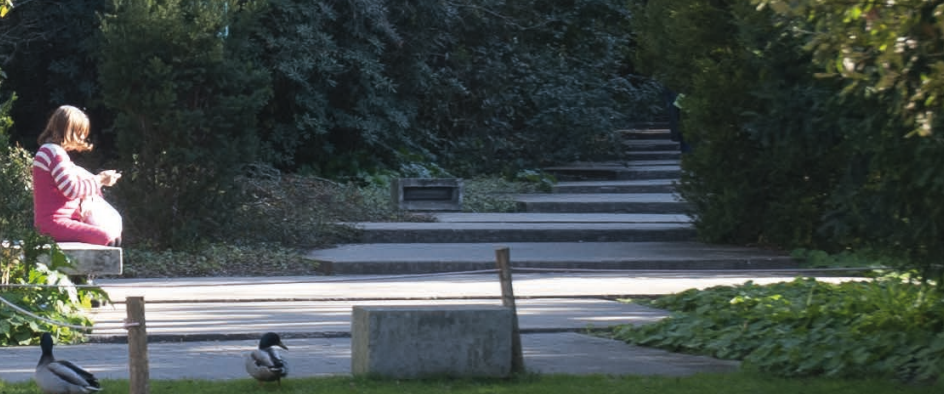

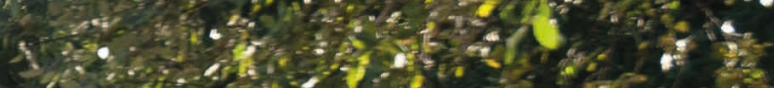

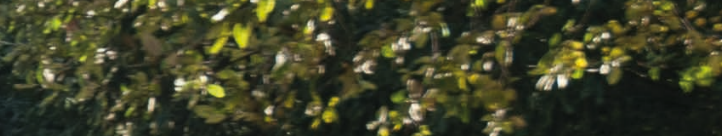


Una di queste è legata alle condizioni di iniquità degli standard abitativi. Che i fenomeni di crescita sempre più veloce dei grandi sistemi urbani portino le fasce economicamente più deboli a determinare insediamenti insalubri, è una consapevolezza condivisa da tutti gli organismi internazionali. È anche parte di una storia dello sviluppo delle città, che però negli ultimi decenni ha avuto un'accelerazione senza precedenti:

The increasing traction of the anti-density movement in the wake of the current outbreak is alarming. [...] Recent studies have debunked these myths [...] instead attributing the spread of the virus to overcrowding due to inequity [...]. Mounting evidence suggests that COVID-19 is primarily transmitted through close contact in enclosed spaces. Internal population density within buildings and, more specifically, within shared rooms inside buildings is what drives this, not the compact urban form of the city. [...] The real issue is the systemic economic inequity that forces lower income people to live in overcrowded conditions, regardless of location. [...]. Vilifying the city is counterproductive. [...] Cities are not at fault. (Grove, 2020)

Grove quindi sottolinea come anche il cosiddetto spillover analizzato da David Quammen (2012), ovvero il passaggio di forme di contagio tra specie diverse inclusa quella umana, sia più frequente nelle aree dove la pressione dell'espansione urbana avviene a contatto, o in sottrazione, di aree con elevata biodiversità originaria. Riporta inoltre come secondo Richard Weller (2017) alcune centinaia di grandi città nel mondo si stiano espandendo proprio all'interno delle aree di maggior biodiversità naturale, creando anche abitudini di contatto e sfruttamento di risorse le cui conseguenze non sono ancora conosciute e analizzate. Quindi che il combinato congiunto dello sviluppo urbano iniquo e della sua collocazione geografica sono un possibile veicolo di diffusione di contagi.

\section{Prospect \& Refuge}

Negli ultimi decenni il dibattito sul ruolo dei sistemi vegetali in ambiente urbano si è esteso in molteplici direzioni e declinazioni sempre più orientate alla costruzione di biodiversità urbane complesse, nelle quali anche il mondo animale possa occupare una posizione significativa. Tra le moltissime articolazioni possiamo evidenziare due contributi sul ruolo del 'selvatico', l'ultimo numero di Jardins, Le Sauvage (2020) e il volume La città selvatica (Metta e Olivetti, 2020), che includono entrambi un discorso sulle possibilità, talvolta conflittuali, di una presenza del selvatico che da occasionale acquisti uno spazio riconosciuto. In maniera simile, anche la rivista $L A+$, con il concorso Creature (2020), ha voluto estendere questo ragionamento alle specie animali non umane, proponendo di fatto un'inclusione riconosciuta di queste nei contesti antopizzati.

Durante il confinamento e subito dopo, giardini e parchi sono stati i luoghi più agognati dai reclusi. Proprio come gli uccelli e gli animali di Appleton, ognuno ha valutato gli spazi della città proprio in questa prospettiva. I sostenitori attuali degli healing gardenes, che studiano gli ortoterapeuti di prima generazione delle kurshalle ottocentesche o delle colonie balilla di mare e montagna, trovano dei riscontri reali per aggiornare quei concetti verso quello attuale di benessere urbano. Le varie riaperture, parziali e non, hanno dimostrato come, pur nella valutazione del rischio, una dotazione generosa di luoghi a disposizione sia un dato essenziale. Ovvero, favorire una città dove sia possibile registrare le proprie vicinanze in maniera più autonoma e individuale, ovvero l'importanza di poter scegliere tra diversi gradi di assembramento o distanziamento.

Riassumendo con Appleton, animali e uomo condividono una condizione per la quale:

la capacità di vedere e la capacità di nascondersi sono entrambe importanti per calcolare le prospettive di sopravvivenza di una creatura [...] La teoria del prospettiva-rifugio postula che, poiché la capacità di vedere senza essere visti è un passo intermedio nella soddisfazione di molte di queste esigenze, la capacità di un ambiente di garantirne il raggiungimento diventa una fonte più immediata di soddisfazione estetica. [...] Dal riconoscimento 


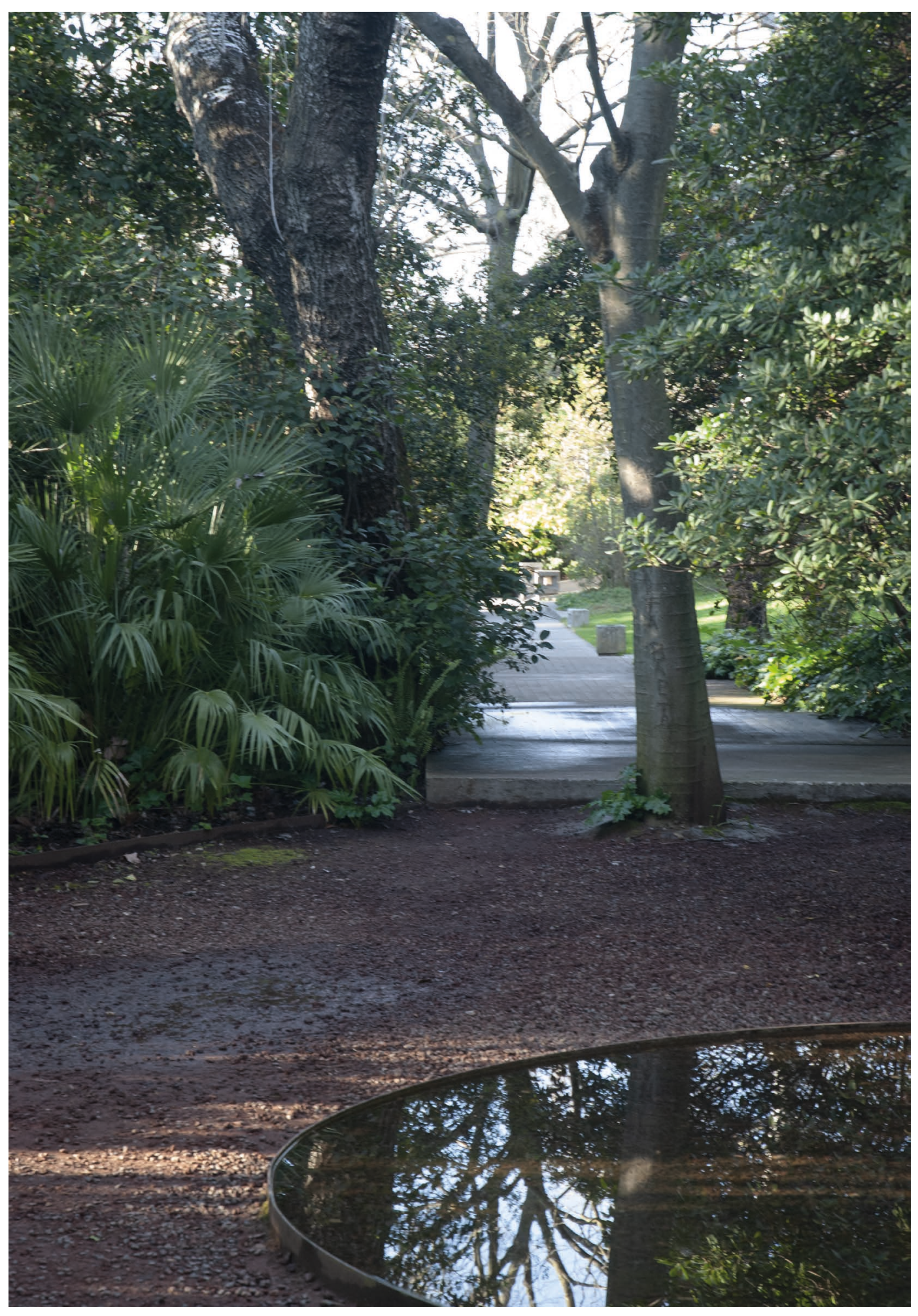


di prospettive e rifugi come basi gemelle della nostra ipotesi può sembrare a prima vista che abbiamo stabilito una dicotomia di opposti [...] 'Vedere' non è chiaramente la stessa cosa di 'non essere visti.' Ma sarebbe piuttosto fallace considerarli come 'opposti' [...] Allo stesso modo, i concetti di prospettiva e rifugio sono antagonisti solo in termini tattici [...] un paesaggio che offre sia una buona opportunità di vedere che una buona opportunità di nascondersi è esteticamente più soddisfacente di uno che non offre nessuno dei due, ma ancora una volta la debolezza nella prospettiva o nel rifugio può essere compensata dalla forza nell'altro (Appelton, 1975).

Pensando e guardando come uccelli, anche alla luce dello spillover e di altro sarebbe utile ripensare alla costruzione di una natura intermedia, tra urbanità e wilderness. Intermedia tra interno ed esterno, diffusa al di là delle fasce urbane, come forme consapevoli di necessità. Quello spazio, forse indeciso, dove il desiderio di 'rifugio' si fonde e dialoga con la necessità di 'prospettiva', non solo percettive ma di opportunità. 


\section{Bibliografia}

Appleton J. 1975, The Experience of Landscape, John Wiley \& Sons, Londra.

Calvino I. 1947, II sentiero dei nidi di ragno, Einaudi, Torino.

Clément C. 2020, Strategia della paura, https://www.quodlibet.it/letture/gilles-cl-nt-strategia-della-paura (12/20).

Dosen A., Ostwald M. 2013, Prospect and Refuge Theory. Constructing a Critical Definition for Architecture and Design, «The International Journal of Design in Society», vol. 6, n. 1 .

Farina A. 2007, Sistemi ambientali ed ecologia cognitiva, in Enciclopedia della Scienza e della Tecnica, Istituto dell'Enciclopedia Italiana G. Treccani, Roma.

Grimal P. 1974, L'art des jardins, Presses Universitaires de France, Parigi. Trad. it.. a cura di M. Magi 2005, L'arte dei giardini, Donzelli, Roma.

Grove M. 2020, Suburban Sprawl Increases the Risk of Future Pandemics, in The Dirt, ASLA, 5 June, https:// dirt.asla.org/2020/05/26/suburban-sprawl-increases-the-risk-of-future-pandemics/ (12/20).

Kroll L. 1999, Tutto è paesaggio, Testo \& Immagine, Torino. Lacoste Y. 2003, De la géopolitique aux paysages, dictionnaire de la géographie, A. Colin, Parigi.

Martella M. 2014, Teodor Ceric, Jardins en temps de guerre, Actes Sud, Parigi. Trad. it. 2015, Giardini in tempo di guerra, Ponte alle Grazie Milano.

Meschiari M. 2020, Cinque domande sullo scenario futuro. Interviste a Walter Siti, Matteo Meschiari, Francesco Guala, «Doppiozero», 8 aprile 2020, https://www.doppiozero. com/materiali/cinque-domande-sullo-scenario-futuro $(12 / 20)$.
Musterd S., Van Zelm I. 2001, Polycentricity, Households and the Identity of Places, «Urban Studies», n. 38, pp. 679696. 10.1080/00420980120035286.

Quammen D. 2014, Spillover. L'evoluzione delle pandemie, Adelphi, Milano.

Racine M. 2015, Des jardiniers pour soigner la médecine, «Jardins», Le Soin, n. 6.

Till F. 2020, Attached to the World: Piet Oudolf on the Garden of Life, "Hauser\&Wirth Magazine», https://www. hauserwirth.com/magazine/29413-attached-world-pietoudolf-garden-life (12/20).

Weller R. J., Hoch C., Huang C. 2017, Atlas for the End of the World, http://atlas-for-the-end-of-the-world.com $(12 / 20)$. 